\title{
Sinus node dysfunction after partial anomalous pulmonary venous connection repair
}

\author{
Carlo Pace Napoleone, MD, ${ }^{\mathrm{a}}$ Elisabetta Mariucci, MD, ${ }^{\mathrm{b}}$ Emanuela Angeli, MD, ${ }^{\mathrm{a}}$ Guido Oppido, MD, ${ }^{\mathrm{a}}$ and \\ Gaetano D. Gargiulo, MD
}

\begin{abstract}
Objective: Repair of partial anomalous pulmonary venous connection to superior vena cava using an internal patch has been described as a potential cause of obstruction at the systemic or pulmonary vein level and of sinus node dysfunction. Our experience with this operation was reviewed.
\end{abstract}

\begin{abstract}
Methods: From 1991 to 2011, 59 patients with a diagnosis of partial anomalous pulmonary venous connection to superior vena cava underwent surgical repair with intracardiac patch rerouting alone (45 patients) or with associated superior vena cava patch enlargement (14 patients). Follow-up evaluation was performed, including electrocardiogram, echocardiogram, electrocardiogram Holter monitor recording, and exercise stress test.
\end{abstract}

\begin{abstract}
Results: There were no early or late deaths and no reoperations at a mean follow-up of $46 \pm 45$ months. All patients were asymptomatic in New York Heart Association class I. Echocardiographic evaluation excluded any obstruction at the pulmonary or systemic vein level. At follow-up, 55 patients $(93 \%)$ presented sinus rhythm and were free from antiarrhythmic medications, 2 patients $(3 \%)$ presented atrial fibrillation, 1 patient $(2 \%)$ presented atrial fibrillation and asymptomatic sinus node dysfunction, and 1 patient $(2 \%)$ presented ectopic atrial rhythm. Electrocardiogram Holter recording demonstrated sinus node dysfunction in 6 of 34 patients $(18 \%)$. Exercise stress test showed chronotropic incompetence in 8 of 27 patients (30\%): All except 1 patient presented sinus rhythm at basal electrocardiogram, and only 4 patients had some evidence of sinus node dysfunction on electrocardiogram Holter recording.
\end{abstract}

Conclusions: Intracardiac repair of partial anomalous pulmonary venous connection can be performed with good results at medium-term follow-up. The rate of sinus node dysfunction or other arrhythmias and obstruction at pulmonary or systemic vein level is comparable to other techniques. Exercise stress test evaluation is the best way to detect asymptomatic sinus node dysfunction. (J Thorac Cardiovasc Surg 2014;147:1594-8)

Sinus venosus atrial septal defects (SV-ASDs), commonly located at the superior vena cava to right atrial (SVC-RA) junction, represent $10 \%$ of all atrial septal defects (ASDs) and are associated in approximately $90 \%$ of cases with partial anomalous pulmonary venous connections (PAPVCs). ${ }^{1,2}$

Numerous surgical procedures have been proposed to correct this anomaly. ${ }^{3}$ The most common operation consists of closing the ASD with a single patch encircling the PAPVC to baffle the pulmonary venous drainage to the left atrium. ${ }^{4}$ When the PAPVC is committed to the superior vena cava (SVC), a postoperative obstruction of the pulmonary veins, the SVC, or both has been described. ${ }^{5}$ A second patch across the SVC-RA junction has been

$\overline{\text { From Pediatric Cardiac Surgery }}{ }^{\mathrm{a}}$ and Pediatric Cardiology, ${ }^{\mathrm{b}}$ S. Orsola-Malpighi Hospital, Bologna Medical School, Bologna, Italy.

Disclosures: Authors have nothing to disclose with regard to commercial support.

Received for publication Jan 16, 2013; revisions received June 19, 2013; accepted for publication July 26, 2013; available ahead of print Sept 23, 2013.

Address for reprints: Carlo Pace Napoleone, MD, Pediatric Cardiac Surgery Unit, S. Orsola-Malpighi Hospital, Via Massarenti 9, 40138 Bologna, Italy (E-mail: pace@aosp.bo.it).

$0022-5223 / \$ 36.00$

Copyright (c) 2014 by The American Association for Thoracic Surgery

http://dx.doi.org/10.1016/j.jtcvs.2013.07.058 proposed to minimize the risk of SVC obstruction but may result in sinoatrial node dysfunction (SND). ${ }^{6}$

The "Warden procedure" was proposed in 1984 as the solution to these problems. ${ }^{7}$ In this technique, the SVC is sectioned above the PAPVC and anastomosed to the right atrial (RA) appendage. SV-ASD closure including the entire SVC orifice is then obtained with a patch. Since then, many reports have stated the superiority of the "Warden procedure" over the single- or 2-patch technique in terms of SND or SVC stenosis at follow-up. ${ }^{8,9}$

At the Bologna Medical School, we have used the singleor 2-patch correction according to the surgeon's preference, usually depending on the distance between the anomalous pulmonary vein connection and the SVC-RA junction or on the residual SVC caliber after PAPVC baffling. To compare our results with the literature, we retrospectively reviewed our experience with particular attention to SND and SVC or pulmonary vein stenosis at follow-up.

\section{MATERIALS AND METHODS Patient Population}

From 1991 to 2010, 59 consecutive patients underwent surgical repair of PAPVC to SVC at the Pediatric Cardiac Surgery Unit of Bologna Medical School, University of Bologna, Italy. The median age at operation was 14 years (range, 0.6-75 years); 33 patients $(57 \%)$ were children aged 


$$
\begin{aligned}
& \text { Abbreviations and Acronyms } \\
& \begin{aligned}
\text { AF } & =\text { atrial fibrillation } \\
\text { ASD } & \text { atrial septal defect } \\
\text { ECG } & =\text { electrocardiogram } \\
\text { PAPVC } & \text { partial anomalous pulmonary venous } \\
& \text { connection } \\
\text { RA } & =\text { right atrial } \\
\text { SN } & \text { sinoatrial node } \\
\text { SND } & \text { sinoatrial node dysfunction } \\
\text { SV- } & =\text { sinus venosus atrial septal defect } \\
\text { ASD } & \\
\text { SVC } & =\text { superior vena cava } \\
\text { SVC- } & \text { superior vena cava to right atrial } \\
\text { RA } &
\end{aligned}
\end{aligned}
$$

less than 18 years. The patients were identified by retrospective review of the surgical database. Informed consent was obtained by all patients or parents. Approval of this study from the research ethics board of the Bologna Medical School was given.

Diagnosis was obtained by echocardiographic evaluation in all patients. Cardiac catheterization with angiography was performed in 16 patients $(27 \%)$ to better define the anatomy of pulmonary veins, and magnetic resonance imaging or computed tomography scans were obtained in 14 patients $(24 \%)$.

\section{Surgical Technique}

All patients underwent surgical correction via median sternotomy. The SVC was cannulated close to the innominate vein, and moderately hypothermic $\left(28^{\circ} \mathrm{C}-34^{\circ} \mathrm{C}\right)$ cardiopulmonary bypass was started. Cardiac arrest was achieved with cold crystalloid cardioplegia and topical cooling with slush saline. A right atriotomy parallel to the interatrial septum was performed and the incision was carried into the SVC, taking care to remain on the right side of the vein, just over the insertion of the anomalous pulmonary veins. Incision was extended distally in the SVC to the inlet of the farthest anomalous pulmonary vein (Figure 1). If SV-ASD was not evident, an adequately sized atrial septectomy was performed with subsequent reconstruction of the endocardial layer with monofilament running suture. Pulmonary vein flow was baffled into the left atrium through the ASD with a heterologous pericardial patch in 48 patients $(81 \%)$ or knit Teflon fabric patch in 11 patients $(19 \%)$. The SVC was closed directly in 45 patients $(76 \%)$. In the remaining 14 patients $(24 \%)$, in whom the anomalous pulmonary veins enter the SVC far from the SVC-RA junction and SVC was considered of inadequate caliber after baffling the anomalous pulmonary veins, heterologous pericardial patch augmentation of the SVC was performed. After removing venous cannulae, the presence of residual pressure gradient across the SVC or anomalous pulmonary veins was evaluated by direct puncture of the vessels.

In 4 patients receiving a direct closure of SVC, a residual gradient greater then $2 \mathrm{~mm} \mathrm{Hg}$ across the SVC-RA junction was disclosed and immediate correction with patch enlargement was undertaken. All patients received oral antiplatelet medication for 6 months.

\section{Follow-up}

A complete follow-up of $46 \pm 45$ months was available. All patients received clinical, echocardiographic and electrocardiogram (ECG) evaluation. To better disclose late SND, 34 patients underwent further investigations: In all of them, 24-hour ECG-Holter monitor recording was obtained, whereas only 27 patients underwent additional exercise stress test evaluation because ages less than 10 years and more than 65 years were considered a bias in obtaining a reliable result.

SND was defined as sinus bradycardia, sinus arrest, sinoatrial block, and paroxysmal supraventricular tachyarrhythmias alternating with periods of bradycardia or even asystole recorded with standard 12-lead ECG or 24-hour ECG-Holter recording. ${ }^{10}$

A minimum heart rate of less than 40 beats/min was considered sinus bradycardia in patients aged more than 16 years, whereas a minimum heart rate of less than 50 beats/min was considered sinus bradycardia in younger patients. A pause more than 3 seconds in adults and more than 3 times the basic cycle length in younger patients was considered pathologic.

To avoid ECG-Holter overdiagnosis of SND in young patients with sinus bradycardia or sinus pauses due to increased vagal tone, we considered ECG-Holter findings consistent with pathologic SND only if even exercise stress test documented chronotropic incompetence in the same patient. Exercise stress test was considered consistent with SND if chronotropic incompetence was documented, such as if the patient was unable to reach a heart rate $85 \%$ or greater of theoretic maximal value for age with maximal effort. ${ }^{10}$

\section{RESULTS}

At the end of the operation, all patients were free of any residual pressure gradient at the SVC or anomalous pulmonary veins level as shown by intracardiac pressure evaluation. There were no early or late deaths.

At follow-up, all patients were asymptomatic and none required reoperation. Echocardiographic evaluation excluded the presence of any residual obstruction or stenosis of the SVC or pulmonary veins and of any shunt at the atrial septal level. None of the patients required a permanent pacemaker for bradyarrhythmias.

Four adult patients $(4 / 59,7 \%$; mean age at operation, 62 \pm 14 years) presented persistent atrial fibrillation (AF) postoperatively. One of them, a 75-year-old man with persistent $\mathrm{AF}$ preoperatively, presented $\mathrm{AF}$ with a slow ventricular rate postoperatively and was discharged without antiarrhythmic medications. The other 3 patients were successfully treated with antiarrhythmic medications or electrical cardioversion postoperatively and discharged in sinus rhythm.

At follow-up, 2 patients, aged 70 and 72 years at operation, developed a first episode of persistent $\mathrm{AF}$ and were treated with rate-control medications. The 75-yearold patient presented persistent $\mathrm{AF}$ with asymptomatic SND (sick sinus syndrome with prevalent bradycardia) at follow-up, but he did not require any antiarrhythmic medications or pacemaker implantation.

One patient, aged 62 years at operation and with postoperatively treated $\mathrm{AF}$, developed an atypical atrial flutter 1 year after operation. He underwent successful transcatheter ablation and was free of antiarrhythmic medications at follow-up.

One young patient, aged 6 years, developed an ectopic atrial tachycardia 6 weeks after surgical operation and was treated successfully with flecainide plus beta-blocker for 6 months with no further recurrency at follow-up.

Briefly, at follow-up with basal 12-lead ECG, 55 patients $(93 \%)$ were in sinus rhythm and free of antiarrhythmic 


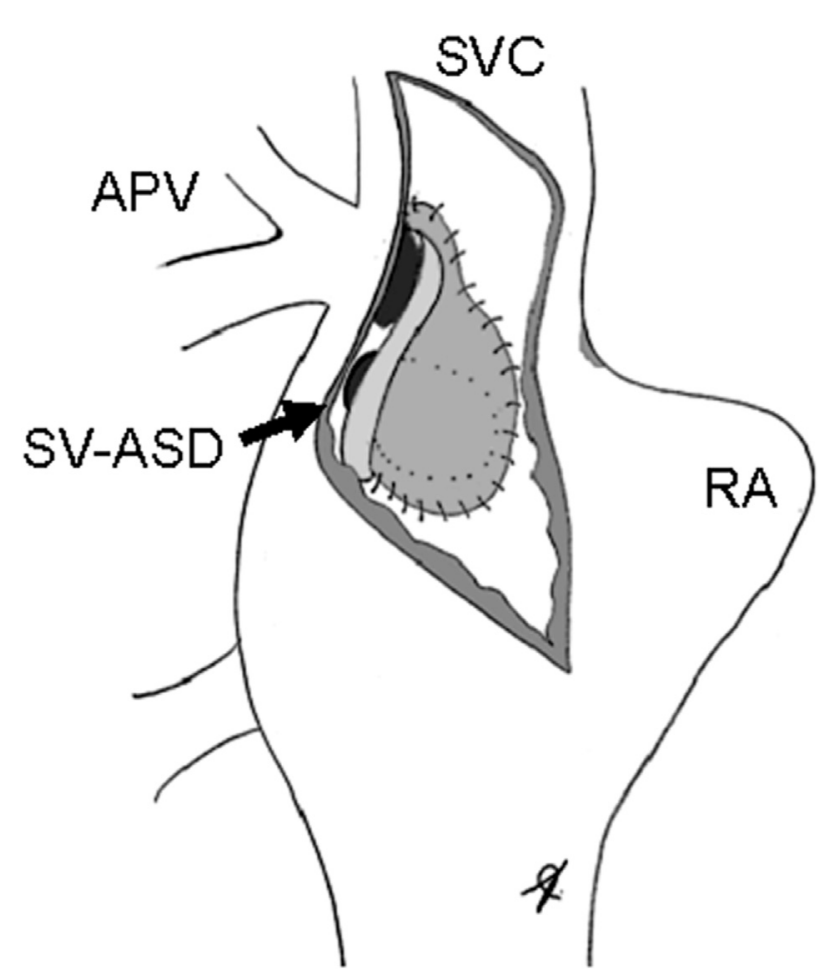

FIGURE 1. Surgical vision of the anomalous pulmonary vein draining in the SVC, clearly evident with an RA carried into the SVC. SV-ASD is used to baffle anomalous pulmonary return to the left atrium. $A P V$, Anomalous pulmonary vein; $R A$, right atrial; $S V-A S D$, sinus venosus atrial septal defect; $S V C$, superior vena cava.

medications, 2 patients $(3 \%)$ were taking rate-control medications for persistent AF, 1 patient $(2 \%)$ presented $\mathrm{AF}$ and asymptomatic sick sinus syndrome with prevalent bradycardia, and 1 patient $(2 \%)$ presented ectopic atrial rhythm.

A 24-hour ECG-Holter monitor recording was obtained in 34 patients, and an exercise stress test was performed in 27 patients at a mean time from operation of $10 \pm 5$ years. Twenty-four-hour ECG-Holter monitor recording demonstrated SND in 6 patients $(6 / 34,18 \%$; mean age, $40 \pm 23$ years). The results are summarized in Table 1 , in which the patient population is divided into 2 groups according to their age, because different cutoff values are considered for patients aged less than or more than 16 years.

The exercise stress test showed chronotropic incompetence in 8 patients $(8 / 27,30 \%$; mean age, $23 \pm 13$ years). All patients except 1 presented sinus rhythm with normal heart rate at basal ECG, and only 4 of them have some evidence of SND at ECG-Holter recording (Table 2).

No correlation was evident between the surgical variables and the incidence of SND at follow-up (Table 3). In particular, age at operation was not statistically significant in affecting SND incidence and surgical strategy (Table 4).
TABLE 1. Results of 24-hour electrocardiogram Holter monitor recording

\begin{tabular}{|c|c|c|}
\hline & Age $<16 y$ & Age $\geq 16$ y \\
\hline Patients & 8 & 26 \\
\hline Age $(y)$ & $13 \pm 3(9-16)$ & $43 \pm 20(17-83)$ \\
\hline Medications* & 0 & $1(4 \%)$ \\
\hline SND-related symptoms & 0 & 0 \\
\hline Mean HR: 24 h (beats/min) & $90 \pm 11(79-108)$ & $74 \pm 8(56-88)$ \\
\hline Minimum HR (beats/min) & $59 \pm 20(27-97)$ & $48 \pm 8(27-60)$ \\
\hline $\begin{array}{l}\text { Minimum } \mathrm{HR}<50 \text { beats } / \mathrm{min} \text {, } \\
\text { patients }(\%)\end{array}$ & $1(12.5 \%)$ & 一 \\
\hline $\begin{array}{l}\text { Minimum } \mathrm{HR}<40 \text { beats/min, } \\
\text { patients }(\%)\end{array}$ & - & $5(19 \%)$ \\
\hline $\begin{array}{l}\text { Sinus arrest or sinoatrial block, } \\
\text { patients }(\%)\end{array}$ & 0 & $1(4 \%)$ \\
\hline \multicolumn{3}{|l|}{ AF/AFL/AT episodes or frequent } \\
\hline SVEB, patients $(\%)$ & 0 & $2(8 \%)$ \\
\hline
\end{tabular}

\section{DISCUSSION}

Although surgical repair of PAPVC has been performed with excellent early outcomes for multiple decades, there have been numerous concerns about postoperative problems related to the obstruction of the SVC or pulmonary veins after baffle redirection of pulmonary venous blood into the left atrium or to anastomotic strictures after implantation of the anomalous pulmonary veins directly on the left atrium. ${ }^{11}$ An additional concern is related to postoperative dysrhythmias that have been reported in multiple series after surgical repair of PAPVC. ${ }^{4,8,9,12}$

Historically, the most common operation for SV-ASD with PAPVC to the RA or RA-SVC junction consists of closing the ASD with a single atrial patch that includes the PAPVC, thereby baffling the pulmonary venous drainage to the left atrium. However, if the PAPVC is committed to the SVC, baffling by a single patch can result in obstruction to the pulmonary veins or the SVC, or both. In this case, the necessity to carry the atrial incision into the SVC up to the anomalous pulmonary vein entrance may result in SND by disrupting the sinoatrial node $(\mathrm{SN})$ or the SN artery. ${ }^{8}$ Moreover, in cases of long incision or small residual SVC caliber, a second patch across the SVC-RA

TABLE 2. Exercise stress test results

\begin{tabular}{lc}
\hline & Mean value \pm SD (range) or $\%$ \\
\hline Patients & 27 \\
Age $(y)$ & $32 \pm 17(11-65)$ \\
Medications* & $1(4 \%)$ \\
Peak effort HR (\% of theoretic value) & $86 \pm 9(65-100)$ \\
Chronotropic incompetence $\dagger$ & $8(30 \%)$ \\
\hline
\end{tabular}

$H R$, Heart rate; $S D$, standard deviation. *Antiarrhythmic or antihypertensive medications with bradycardic effects. †Unable to reach $\mathrm{HR} \geq 85 \%$ of theoretic value at peak effort, excluded patients with medications. 
TABLE 3. Correlation between surgical and clinical variables and postoperative sinus node dysfunction

\begin{tabular}{lccc}
\hline & SND & SR & $\boldsymbol{P}$ \\
\hline Patients & 8 & 51 & \\
Age (y) & $13.1 \pm 12.1$ & $25.7 \pm 22.7$ & NS \\
Surgically created ASD & $1 / 8(12.5 \%)$ & $9 / 51(17.6 \%)$ & NS \\
2-patch technique & $1 / 8(12.5 \%)$ & $13 / 51(25.4 \%)$ & NS \\
Baffle material & & & \\
$\quad$ Pericardial & $5 / 8(62.5 \%)$ & $43 / 51(84.3 \%)$ & NS \\
$\quad$ Teflon & $3 / 8(37.5 \%)$ & $8 / 51(15.7 \%)$ & \\
Postoperative rhythm problem & $0 / 8$ & $8 / 51(15.7 \%)$ & NS \\
\hline
\end{tabular}

$A S D$, Atrial septal defect; $N S$, not significant; $S N D$, sinus node dysfunction; $S R$, sinus rhythm.

junction minimizes the risk of SVC obstruction but can distort this region, leading to $\mathrm{SN}$ malfunction.

In 1984, Warden and colleagues ${ }^{7}$ described a procedure used in 15 patients with PAPVC into the SVC. The SVC is transected above the PAPVC and anastomosed to the RA appendage, followed by ASD patch closure that includes the entire SVC orifice. The Warden procedure provides unobstructed drainage of the SVC and pulmonary veins and virtually eliminates the problem of SND that was frequently seen with the 2-patch repair. Many reports described a near-absent incidence of SND early postoperatively and during follow-up. ${ }^{13}$

Stewart and colleagues ${ }^{8}$ demonstrated that the use of the 2-patch technique was associated with a low incidence of SVC or pulmonary vein stenosis; however, the incidence of SND was significant $(55 \%)$. They reported no SND in the 5 patients who underwent the Warden procedure, presumably because the procedure eliminates any incisions near the SN or SN artery, but the follow-up time was shorter for the Warden group. ${ }^{8}$

In our experience, we failed to demonstrate any correlation between the 2-patch technique and SND (Table 3). This can be explained also with the selected use of this technique that was limited to only 14 patients.

Shahriari and colleagues ${ }^{9}$ reported the results of 54 patients with SV-ASD repaired at Riley Children's Hospital, 27 of whom had a single-patch technique, 12 of whom had a 2-patch technique, and 13 of whom had a Warden procedure. Although there was a low rate of arrhythmias in the entire series, $100 \%$ of the patients who underwent Warden procedures remained in normal sinus rhythm.

Stewart and coworkers ${ }^{4}$ reported 15 patients with SV-ASD and PAPVC to the SVC repaired with a single-

TABLE 4. Correlation between age and surgical variables

\begin{tabular}{lcccccc}
\hline & \multicolumn{2}{c}{ Yes } & & \multicolumn{2}{c}{ No } & \\
\cline { 2 - 3 } \cline { 5 - 6 } & No. & Mean age & & No. & Mean age & $\boldsymbol{P}$ \\
\hline Surgically created ASD & 10 & $20.9 \mathrm{y}$ & & 49 & $24.7 \mathrm{y}$ & NS \\
2-patch technique & 14 & $18.6 \mathrm{y}$ & & 45 & $25.7 \mathrm{y}$ & NS \\
\hline
\end{tabular}

$A S D$, Atrial septal defect; $N S$, not significant. patch technique, which included an incision from the atrial appendage superiorly along the SVC to the level of the PAPVC, thus directly across the SN. They noted that $40 \%$ of the patients had early SND. Of note, they found that all of these postoperative rhythm problems resolved before hospital discharge. ${ }^{4}$

A more recent report compared the single-patch, doublepatch, and Warden technique in 54 patients. The incidence of low atrial or junctional rhythm was significantly higher with the double-patch repair (55\%) compared with the single-patch $(24 \%)$ and Warden $(0 \%)$ repairs. No differences were evidenced in terms of SVC stenosis among the 3 techniques.

Many reports in the literature seem to corroborate the finding that the patch technique, and above all the doublepatch technique, is associated with a high incidence of SND on short- and mid-term follow-ups, whereas the Warden procedure essentially eliminates SN injury and thus SND.

It is important to underline that in all these reports the SND diagnosis was based on symptoms and 12-lead ECG. As demonstrated by our experience, the actual SND prevalence can be largely underestimated. Limiting analysis to symptoms and ECG evaluation would have disclosed only 2 patients with SND in our experience, with a prevalence of $3 \%$ (2/59 patients) at a mean term followup, a result that can be easily compared with that of Warden technique reported in the literature. ${ }^{4-6,8,9,11-13}$

SND diagnosis was based on 24-hour Holter evaluation at follow-up by Agarwal and colleagues ${ }^{14}$ who reported no incidence of SND among 58 patients undergoing the Warden operation. In our experience, this value was greater because 24-hour Holter disclosed 6 of 34 patients (18\%) who reached the criteria for SND.

Although many experienced clinicians claim to recognize SND in individual patients, no single metric has been established as a diagnostic standard. ${ }^{10}$ Regardless, we think that the most accurate way is to perform a more comprehensive evaluation with ECG, ECG-Holter, and exercise stress test because of the intermittent nature of the SND episodes. In this way, we observed a different incidence of SND (8/27 patients, $30 \%)$, which we considered the real incidence of rhythm problems after single- or doublepatch repair of this heart disease.

To the best of our knowledge, our report is the first to apply a global evaluation of SND in patients undergoing operation for PAPVC. Without this approach, all other reports may underestimate the incidence of SND and, for this reason, are not comparable to our results. We hope that our work will be shared by other researchers to disclose the correct incidence of SND after PAPVC surgical correction.

\section{Study Limitations}

This report presents the well-known limitations of a retrospective and nonrandomized study. We are aware 
that the incidence of chronotropic incompetence can be overestimated if the test is not driven to maximal effort, and even ECG-Holter can lead to overdiagnosis of SND in young patients with sinus bradycardia or sinus pauses due to increased vagal tone. Regardless, we believe that SND can remain undetected without these tests. We did our best to perform maximal effort and symptom-driven exercise tests and tried to avoid ECG-Holter SND overdiagnosis by performing a complete evaluation of all clinical data (eg, considering ECG-Holter findings consistent with pathologic SND only if even exercise stress test documented chronotropic incompetence in the same patient).

\section{CONCLUSIONS}

Our data report a short-term incidence of SND with the single- or double-patch technique almost comparable to the reported experiences with the Warden procedure. Nevertheless, the long-term incidence of SND after PAPVC repair can be approximately $20 \%$ to $30 \%$ and may warrant longterm follow-up with ECG-Holter monitor recordings and exercise stress test even if the patients are asymptomatic, because SND can be demonstrated only with an extensive and accurate evaluation. To define the true late effects on rhythm and to better compare the 2 techniques, further studies reporting long-term follow-up of patients receiving the Warden technique with ECG-Holter monitor recordings and exercise stress test are necessary.

\section{References}

1. Lewis FJ. High defects of the atrial septum. J Thorac Surg. 1958;36:1-11.

2. Cooley DA, Latson JR, Keats AS. Surgical considerations in repair of ventricular and atrial septal defects utilizing cardiopulmonary bypass; experience with 104 cases. Surgery. 1958;43:214-25.
3. Takahashi H, Oshima Y, Yoshida M, Yamaguchi M, Okada K, Okita Y. Sinus node dysfunction after repair of partial anomalous pulmonary venous connection. J Thorac Cardiovasc Surg. 2008;136:329-34.

4. Stewart S, Alexson C, Manning J. Early and late results of repair of partial anomalous pulmonary venous connection to the superior vena cava with a pericardial baffle. Ann Thorac Surg. 1986;41:498-501.

5. Iyer AP, Somanrema K, Pathak S, Manjunath PY, Pradhan S, Krishnan S. Comparative study of single- and double-patch techniques for sinus venosus atrial septal defect with partial anomalous pulmonary venous connection. J Thorac Cardiovasc Surg. 2007;133:656-9.

6. Said SM, Burkhart HM, Schaff HV, Cetta F Jr, Phillips SD, Barnes RD, et al. Single-patch, 2-patch, and caval division techniques for repair of partial anomalous pulmonary venous connections: does it matter? J Thorac Cardiovasc Surg. 2012;143:896-903.

7. Warden HE, Gustafson RA, Tarnay TJ, Neal WA. An alternative method for repair of partial anomalous pulmonary venous connection to the superior vena cava. Ann Thorac Surg. 1984;38:601-5.

8. Stewart RD, Bailliard F, Kelle AM, Backer CL, Young L, Mavroudis C. Evolving surgical strategy for sinus venosus atrial septal defect: effect on sinus node function and late venous obstruction. Ann Thorac Surg. 2007;84: 1651-5.

9. Shahriari A, Rodefeld MD, Turrentine MW, Brown JW. Caval division technique for sinus venosus atrial septal defect with partial anomalous pulmonary venous connection. Ann Thorac Surg. 2006;81:224-9.

10. Epstein AE, DiMarco JP, Ellenbogen KA, Estes NA 3rd, Freedman RA, Gettes LS, et al. ACC/AHA/HRS 2008 Guidelines for Device-Based Therapy of Cardiac Rhythm Abnormalities: a report of the American College of Cardiology/American Heart Association Task Force on Practice Guidelines (Writing Committee to Revise the ACC/AHA/NASPE 2002 Guideline Update for Implantation of Cardiac Pacemakers and Antiarrhythmia Devices) developed in collaboration with the American Association for Thoracic Surgery and Society of Thoracic Surgeons. J Am Coll Cardiol. 2008;51:e1-62.

11. Alsoufi B, Cai S, Van Arsdell GS, Williams WG, Caldarone CA, Coles JG. Outcomes after surgical treatment of children with partial anomalous pulmonary venous connection. Ann Thorac Surg. 2007;84:2020-6.

12. Trusler GA, Kazenelson G, Freedom RM, Williams WG, Rowe RD. Late results following repair of partial anomalous pulmonary venous connection with sinus venosus atrial septal defect. J Thorac Cardiovasc Surg. 1980;79: 776-81.

13. Okonta KE, Agarwal V. Does Warden's procedure reduce sinus node dysfunction after surgery for partial anomalous pulmonary venous connection? Interact Cardiovasc Thorac Surg. 2012;14:839-42.

14. Agarwal V, Okonta KE, Abubakar U, Gichuhi S. Impact of Warden's procedure on the sinus rhythm: our experience. Heart Lung Circ. 2011;20:718-21. 